EUROPEAN JOURNAL OF PURE AND APPLIED MATHEMATICS

Vol. 15, No. 1, 2022, 199-206

ISSN 1307-5543 - ejpam.com

Published by New York Business Global

\title{
Weakly Covering Spaces in Coc-open Sets
}

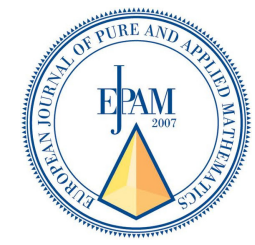

Fuad A. Abushaheen ${ }^{1, *}$, Fadi Alrimawi ${ }^{2}$

${ }^{1}$ Basic Science Department, Middle East University, Amman, Jordan

2 Department of Basic Sciences, Al-Ahliyya Amman University, Amman, Jordan

\begin{abstract}
In this paper, we define several types of weakly covering spaces concerning coc-open sets, we study some implications between these weakly covering spaces. Also, we give several product theorems under coc-almost open function.
\end{abstract}

2020 Mathematics Subject Classifications: 54B05, 54B10, 54D20

Key Words and Phrases: coc-weakly-compact, coc-almost-compact, coc-nearly-compact, cocR-compact

\section{Introduction and Preliminaries}

It is known that covering spaces play very important role in topology. After the concept of compactness defined, many authors studied the relations between this notion and other topological and analytical concepts. Moreover the topologists gave many different generalizations of compactness stands on the types of covers, open sets and subcovers. The discussion about these covering spaces is still a rich area to study in topology.

Also, the idea of giving weaker and stronger forms of open sets is also a hot topic in research. For example semi-open set [9], pre-open [10], no where dense [12] and many more.

In [8], a regular-open set is a set that equals to interior of its closure or equivalently , a subset $A$ of a topological space $X$ is regular open if $\operatorname{int}(\bar{A})=A$ where the closure of $A$ and the interior of $A$ are denoted by $\bar{A}$ and $\operatorname{int}(A)$, respectively. The complement of a regular open said is said to be regular closed, or $A=\overline{\operatorname{int}(A)}$. It is clearly that regularly open is an open set. For further studied in regular open spaces in covering spaces and separation axioms, see [1-5].

In 2012, Al-Ghour and Samarah [7] defined a new type of open sets called coc-compact, a subset $A$ of a topological space $X$ is called coc-open set if $A$ is a union of sets of the form $V-C$, where $V$ is open set and $C$ is a compact subset of $X$. Also, they proved that the family of all coc-open sets of a topological space $(X, \tau)$ forms a topology on $X$ finer than $\tau$. For more results about coc-open subsets, see [11].

*Corresponding author.

DOI: https://doi.org/10.29020/nybg.ejpam.v15i1.4248

Email addresses: fshaheen@meu.edu.jo (F.A. Abushaheen), f.rimawi@ammanu.edu.jo (F. Alrimawi) 
In this paper, no separation axioms to be assumed. A space $X$ means nonempty sets with topology. $\omega_{0}$ denoted the cardinal number of $\mathbb{N}$, for a subset $A$ of $X, \bar{A}^{c o c}$ and $i n t_{c o c}(A)$ will denote the closure of $A$ and the interior of $A$ in $\tau^{k}$, respectively. For notation not mention here, we refer the reader to [6].

The following definitions are important for our paper.

Definition 1. [7] A subset $A$ of a topological space $X$ is called co-compact open set (notation: coc-open) if for every $x \in A$, there exists an open set $U \subseteq X$ and a compact subset $K$ of $X$ such that $x \in U-K \subseteq A$. The complement of a coc-open subset is called coc-closed. The family of all coc-open subsets of a topological space $(X, \tau)$ will be denoted by $\tau^{k}$.

Definition 2. [11] A topological space $X$ is called coc- $T_{2}$-space if and only if for all $x, y \in X$ with $x \neq y \in X$, there exist $U, V \in \tau^{k}$ such that $x \in U, y \in V$ with $U \cap V=\phi$.

Definition 3. A subset $A$ of a topological space $X$ is coc-regular open if int coc $\left(\bar{A}^{c o c}\right)=A$, the complement of a coc-regular open is said to be coc-regular closed, or $A=\overline{\operatorname{int}}_{c o c}(A){ }^{c o c}$.

Definition 4. [7] $A$ family $\mathcal{U} \subseteq X$ is coc-open cover of $X$ if $\mathcal{U}$ covers $X$ and $\mathcal{U}$ is a subfamily of $\tau^{k}$.

Definition 5. A coc-open cover $\mathcal{U}=\left\{U_{\alpha} \mid \alpha \in \Delta\right\}$ of $X$ is called coc-regular cover, if for each $\alpha \in \Delta$ there exists a coc-regular closed set $F_{\alpha}$ such that $F_{\alpha} \subseteq U_{\alpha}$ and $X=$ $\bigcup\left\{\right.$ int $\left._{c o c}\left(F_{\alpha}\right) \mid \alpha \in \Delta\right\}$.

Definition 6. [7] A space $(X, \tau)$ is coc-compact if every coc-open cover has a finite subcover.

Definition 7. A space $(X, \tau)$ is coc-almost-compact if every coc-open cover of $X$ has a finite collection such that the coc-closure of the union is $X$.

Definition 8. A space $(X, \tau)$ is coc-weakly-compact if every coc-regular cover of $X$ has a finite collection such that the coc-closure of the union is $X$.

Definition 9. A space $(X, \tau)$ is coc-nearly-compact if every coc-open cover of $X$ by cocregular open sets has a finite subcover.

Clearly the following implications are true for a topological space $X$ :

coc-compact $\Rightarrow$ coc-nearly compact $\Rightarrow$ coc-almost compact $\Rightarrow$ coc-weakly compact.

The following definition gives a coc-covering space between coc-almost-compact and coc-weakly-compact spaces.

Definition 10. A space $X$ is called coc-R-compact if every coc-regular cover of $X$ has a finite subcover.

It is obvious that coc-R-compactness implies coc-weak-compactness.

Theorem 1. A coc-almost-compact space $X$ is coc-R-compact-space.

Proof. Let $\mathcal{U}=\left\{U_{\alpha} \mid \alpha \in \Delta\right\}$ be a coc-regular cover of $X$ with $F_{\alpha} \subseteq U_{\alpha}$ and $X=$ $\bigcup\left\{\operatorname{int}_{c o c}\left(F_{\alpha}\right) \mid \alpha \in \Delta\right\}$, but $X$ is coc-almost-compact, so there exists a finite subset $\Delta_{0}$ of $\Delta$ such that $X=\bigcup\left\{{\overline{\text { int }} \operatorname{coc}\left(F_{\alpha}\right)}^{c o c} \mid \alpha \in \Delta_{0}\right\}$, so $X=\bigcup\left\{U_{\alpha} \mid \alpha \in \Delta_{0}\right\}=\overline{\bigcup\left\{U_{\alpha} \mid \alpha \in \Delta_{0}\right\}}{ }^{c o c}$, hence $X$ is coc-R-compact-space. 


\section{Main Results}

In the beginning of this section, we explore some results concerning subspaces via coc-weakly covering spaces.

Definition 11. A subset $S$ of a space $X$ is said to be coc-weakly-compact subset if $S$ is coc-weakly-compact subspace of $X$.

Definition 12. A subset $S$ of a space $X$ is said to be coc-weakly-compact relative to $X$, if for each cover $\left\{V_{\alpha} \mid \alpha \in \Delta\right\}$ of $S$ by coc-open sets of $X$ satisfying the following condition $(* *)$, there exists a set $\Delta_{0} \subseteq \Delta$ such that $S \subseteq \bigcup\left\{{\overline{V_{\alpha}}}^{c o c}\left|\alpha \in \Delta_{0} \subseteq \Delta,\right| \Delta_{0} \mid<\omega_{0}\right\}$, where the condition $(* *)$ is :

"for each $\alpha \in \Delta$, there exists a nonempty coc-regular closed set $F_{\alpha}$ such that $F_{\alpha} \subseteq V_{\alpha}$ and $S \subseteq \bigcup\left\{\operatorname{int}_{c o c}\left(F_{\alpha}\right) \mid \alpha \in \Delta\right\} " \quad(* *)$.

Theorem 2. If $A$ is a coc-weakly-compact subspace of a space $X$, then $A$ is coc-weaklycompact relative to $X$.

Proof. Let $\left\{V_{\alpha} \mid \alpha \in \Delta\right\}$ be a cover of $A$ by coc-open sets of $X$ satisfying the condition $(* *)$, for each $\alpha \in \Delta$ there exists a non empty coc-regular closed set $F_{\alpha}$ such that $F_{\alpha} \subseteq V_{\alpha}$

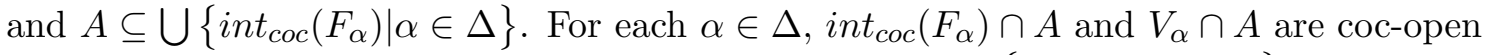
subsets in $A$ and $F_{\alpha} \cap A$ is coc-closed in $A$, so the family $\left\{V_{\alpha} \cap A \mid \alpha \in \Delta\right\}$ is coc-open cover of $A$, therefore for each $\alpha \in \Delta$, we have

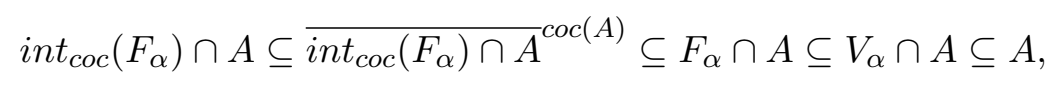

moreover

$$
A=\bigcup\left\{i n t_{c o c}\left(F_{\alpha}\right) \cap A \mid \alpha \in \Delta\right\}
$$

and

$$
\operatorname{int}_{\operatorname{coc}}\left(F_{\alpha}\right) \cap A \subseteq i n t_{c o c}(A)\left({\overline{i n t_{c o c}\left(F_{\alpha} \cap A\right)}}^{\operatorname{coc}(A)} .\right.
$$

Now, the set ${\overline{i n t} \operatorname{coc}\left(F_{\alpha} \cap A\right)}^{\operatorname{coc}(A)}$ is coc-regular closed in $A$ and $\left\{V_{\alpha} \cap A \mid \alpha \in \Delta\right\}$ is a coc-regular cover of $A$, so there exists a finite subset $\Delta_{0} \subseteq \Delta$ such that $A=\bigcup\left\{{\overline{V_{\alpha} \cap A}}^{\operatorname{coc}(A)} \mid \alpha \in \Delta\right\}$, but ${\overline{V_{\alpha} \cap A}}^{\operatorname{coc}(A)} \subseteq{\overline{V_{\alpha}}}^{\operatorname{coc}(A)}$ for each $\alpha \in \Delta_{0}$, therefore $A \subseteq \bigcup\left\{\overline{V_{\alpha}}{ }^{c o c} \mid \alpha \in \Delta_{0}\right\}$, hence the result.

Corollary 1. If $A$ is coc-compact (coc-almost-compact, coc-nearly-compact) subspace of $X$, then $A$ is coc-weakly-compact relative to $X$.

Theorem 3. If every coc-regular closed subset of a space $X$ is coc-weakly compact relative to $X$, then $X$ is coc-weakly compact.

Proof. Let $\left\{V_{\alpha} \mid \alpha \in \Delta\right\}$ be a coc-regular cover of $X$, for each $\alpha \in \Delta$, there exists nonempty coc-regular set such that $F_{\alpha} \subseteq V_{\alpha}$ and $X=\bigcup\left\{i n t_{c o c}\left(F_{\alpha}\right) \mid \alpha \in \Delta\right\}$. For $\alpha_{0} \in \Delta$ let $K=X-\operatorname{int}_{c o c}\left(F_{\alpha_{0}}\right)$, then $K$ is a coc-regular closed and $K \subseteq \bigcup\left\{\right.$ int $_{c o c}\left(F_{\alpha}\right) \mid \alpha \in$ 
$\left.\Delta-\left\{\alpha_{0}\right\}\right\}$, so $\mathcal{V}^{*}=\left\{V_{\alpha} \mid \alpha \in \Delta-\left\{\alpha_{0}\right\}\right\}$ is a cover of $K$ by coc-open sets of $X$ satisfying condition (**) and hence for some finite set $\Delta_{0} \subseteq \Delta$ we have $K \subseteq \bigcup\left\{{\overline{V_{\alpha}}}^{c o c} \mid \alpha \in \Delta_{0}\right\}$, thus

$$
X=K \cup i n t_{c o c}\left(F_{\alpha_{0}}\right) \subseteq \bigcup\left\{{\overline{V_{\alpha}}}^{c o c} \mid \alpha \in \Delta_{0} \cup\left\{\alpha_{0}\right\}\right\},
$$

so $X$ is coc-weakly compact.

Corollary 2. If every proper coc-regular closed subset of a space $X$ is coc-weakly compact, then $X$ is coc-weakly compact.

Proof. Clearly.

Corollary 3. If every proper coc-regular closed subset of a space $X$ is coc-compact (cocalmost-compact, coc-nearly-compact) subspace, then $X$ is coc-weakly compact.

Theorem 4. If a subset $A$ of a coc-weakly-compact space $X$ is coc-clopen, then $A$ is coc-weakly-compact relative to $X$.

Proof. Let $\mathcal{U}_{A}=\left\{U_{\alpha} \mid \alpha \in \Delta\right\}$ be a cover of $A$ by coc-open sets of $X$ satisfying condition (**), hence the family $\left\{U_{\alpha} \mid \alpha \in \Delta\right\} \cup\{X-A\}$ is a coc-regular cover of $X$, but $X$ is coc-weakly-compact, so there exists a finite set $\Delta_{0} \subseteq \Delta$ such that

$$
X \cup\left(\bigcup_{\alpha \in \Delta_{0}}{\overline{V_{\alpha}}}^{c o c}\right) \cup\left(\overline{X-A}{ }^{c o c}\right)=\left(\bigcup_{\alpha \in \Delta_{0}}{\overline{V_{\alpha}}}^{c o c}\right) \cup(X-A),
$$

therefore $A \subseteq \bigcup_{\alpha \in \Delta_{0}}{\overline{V_{\alpha}}}^{c o c}$, therefore $A$ is coc-weakly-compact relative to $X$.

The next definition makes our coc-weakly covering spaces equivalent.

Definition 13. A space $X$ is said to be coc-almost regular if for each coc-regular closed $F$ and $x \in X-F$, there exist disjoint coc-open sets $U, V$ such that $F \subseteq U$ and $x \in V$.

Theorem 5. If a space $X$ is coc-weakly-compact and coc-almost-regular, then $X$ is cocnearly-compact.

Proof. Let $\mathcal{U}=\left\{U_{\alpha} \mid \alpha \in \Delta\right\}$ be coc-regular open cover of $X$, so for each $x \in X$, there exists $\alpha(x) \in \Delta$ such that $x \in U_{\alpha(x)}$, but $X$ is coc-almost regular space, so there exist coc-regular open sets $V_{\alpha(x)}$ and $W_{\alpha(x)}$ such that

$$
x \in V_{\alpha(x)} \subseteq{\overline{V_{\alpha(x)}}}^{c o c} \subseteq W_{\alpha(x)} \subseteq{\overline{W_{\alpha(x)}}}^{c o c} \subseteq U_{\alpha(x)},
$$

and clearly the family $\left\{W_{\alpha(x)} \mid \alpha(x) \in \Delta\right\}$ is coc-regular cover of $X$, so there exists a finite elements $x_{1}, x_{2}, \ldots, x_{n} \in X$ such that

$$
X=\bigcup_{i=1}^{n}{\overline{W_{\alpha\left(x_{i}\right)}}}^{c o c} \subseteq \bigcup_{i=1}^{n} U_{\alpha\left(x_{i}\right)},
$$

therefore $X$ is coc-nearly-compact space. 
Corollary 4. A coc-almost-regular space is coc-weakly-compact if and only if it is cocnearly-compact.

Proof. Obvious.

Corollary 5. A coc- $T_{2}$-space is coc-nearly-compact if and only if it is coc-weakly compact and coc-almost regular.

Proof. $(\Leftarrow)$ Clearly.

$(\Rightarrow)$ Enough to show that $X$ is coc-almost regular. Let $A$ be a coc-regular closed set and $X-A$, assume $y \in A$. Since $X$ is coc- $T_{2}$-space, there exists a coc-open set $V_{y}$ contains $y$ with $x \notin \bar{V}_{y}^{c o c}$. The family $\mathcal{V}=\left\{V_{y} \mid y \in A\right\} \cup\{X-A\}$ is coc-regular open cover of $X$, so there exists a finite subcover $\mathcal{V}^{*}$ of $X$, let $V=\bigcup\left\{V_{\alpha} \in \mathcal{V}^{*} \mid V_{\alpha} \cap A \neq \phi\right\}$, then $V$ is coc-open subset of $X$ and $A \subseteq V$, therefore for each $V_{\alpha} \in \mathcal{V}^{*}$ we have $V_{\alpha} \subseteq V_{y}$ and $x \notin \bar{V}^{c o c}$, hence $X$ is coc-almost-regular.

Corollary 6. The following are equivalent for a coc-almost-regular space:

(i) $X$ is coc-nearly-compact,

(ii) $X$ is coc-almost-compact,

(iii) $X$ is coc-R-compact,

(iv) $X$ is coc-weakly-compact.

Proof. The prove comes from Theorems 5 and 1.

Corollary 7. Let $\left(X, \tau^{k}\right)$ be a $T_{3}$-space and $X$ is coc-weakly-compact, then $X$ is coccompact space.

Proof. The result comes from Theorem 5 and the fact that every $T_{3}$-space in $\left(X, \tau^{k}\right)$ is coc-almost regular .

Definition 14. A function $f: X \rightarrow Y$ is said to be coc-almost open function if $f(U) \subseteq$ int $_{\text {coc }}\left(\overline{f(U)}^{c o c}\right)$ for every coc-open set $U$ of $X$.

Lemma 1. A function $f: X \rightarrow Y$ is coc-almost open if and only if $f^{-1}\left(\bar{V}^{c o c}\right) \subseteq \bar{f}^{-1}(V){ }^{c o c}$ for each coc-open subset $V$ of $Y$.

Proof. $(\Rightarrow)$ : Let $V$ be a coc-open subset of $Y$ with $x \in f^{-1}\left(\bar{V}^{c o c}\right)$ and $U$ is a coc-open subset of $X$ contains $x$, then $f(x) \in f(U) \cap \bar{V}^{c o c} \subseteq i n t_{c o c}\left(\bar{U}^{c o c}\right) \cap \bar{V}^{c o c}$, so $V \cap i n t_{c o c}\left(\bar{U}^{c o c}\right) \neq$ $\phi$ and $U \cap f^{-1}(V) \neq \phi$, therefore $x \in{\overline{f^{-1}(V)}}^{c o c}$.

$(\Leftarrow)$ : If $f$ is not coc-almost open function, so there is a coc-open set $U$ with $f(U) \nsubseteq$ int $_{c o c} f\left(\bar{U}^{c o c}\right)$. Let $V=Y-\overline{f(U)}^{c o c}$, then $V \cap f(U)=\phi$, but $V \subseteq Y-f(U)$, so $\bar{V}^{c o c} \cap f(U) \neq$ $\phi$, so $U \cap f^{-1}\left(\bar{V}^{c o c}\right) \neq \phi$ and $U \cap{\overline{f^{-1}(V)}}^{c o c} \neq \phi$, hence $U \cap f^{-1}(V) \neq \phi$ which contradicts the fact that $f(U) \cap V=\phi$, hence the result. 
Definition 15. A function $f: X \rightarrow Y$ is called coc-perfect function if it is surjective, coc-closed and $f^{-1}(y)$ coc-compact.

Theorem 6. Let $f: X \rightarrow Y$ be a coc-almost-open coc-perfect function. If $K$ is a cocweakly-compact relative to $Y$, then $f^{-1}(K)$ is coc-weakly-compact relative to $X$.

Proof. Let $K$ be coc-weakly-compact relative to $Y$ and $\left\{U_{\alpha} \mid \alpha \in \Delta\right\}$ any cover of $f^{-1}(K)$ by coc-open sets of $X$ satisfies condition $(* *)$. For each $\alpha \in \Delta$ there exists a coc-regular closed set $F_{\alpha}$ of $X$ such that $F_{\alpha} \subseteq U_{\alpha}$ and $f^{-1}(K) \subseteq \bigcup\left\{\operatorname{int}_{c o c}\left(F_{\alpha}\right) \mid \alpha \in \Delta\right\}$.

Now for each $y \in Y$, we have

$$
f^{-1}(y) \subseteq \bigcup\left\{i n t_{c o c}\left(F_{\alpha}\right)|\alpha \in \Delta(y),| \Delta(y) \mid<\omega_{0}\right\}
$$

and

$$
f^{-1}(y) \subseteq \bigcup_{\alpha \in \Delta(y)} i n t_{c o c}\left(F_{\alpha}\right) \subseteq \bigcup_{\alpha \in \Delta(y)} U_{\alpha}
$$

Define

$$
\begin{aligned}
& G_{y}=Y-f\left(X-\bigcup\left\{\operatorname{int}_{c o c}\left(F_{\alpha}\right)|\alpha \in \Delta(y),| \Delta(y) \mid<\omega_{0}\right\}\right), \\
& H_{y}=Y-i n t_{c o c}\left(\frac{f\left(X-\bigcup\left\{F_{\alpha}|\alpha \in \Delta(y),| \Delta(y) \mid<\omega_{0}\right\}\right)}{c o c},\right.
\end{aligned}
$$

and

$$
V_{y}=V-f\left(X-\bigcup\left\{U_{\alpha}|\alpha \in \Delta(y),| \Delta(y) \mid<\omega_{0}\right\}\right),
$$

clearly $G_{y}$ and $V_{y}$ are coc-open subsets in $Y, H_{y}$ is coc-regular closed with $y \in G_{y} \subseteq$ $H_{y} \subseteq V_{y}$, therefore the family $\mathcal{V}=\left\{V_{y} \mid y \in K\right\}$ is a cover of $K$ by coc-open subsets of $Y$ satisfies condition $(* *)$, but $K$ is a coc-weakly-compact relative to $Y$, so there exists set $y_{1}, y_{2}, \ldots, y_{n} \in Y$ of such that

$$
K \subseteq \bigcup\left\{{\overline{V_{y_{i}}}}^{c o c} \mid i=1,2, \ldots, n\right\},
$$

but $f^{-1}\left({\overline{V_{y}}}^{c o c}\right) \subseteq{\overline{f^{-1}\left(V_{y}\right)}}^{c o c}$ for all $y \in Y$, so

$$
f^{-1}\left(V_{y}\right) \subseteq \bigcup\left\{U_{\alpha} \mid \alpha \in \Delta(y)\right\},
$$

therefore

$$
f^{-1}(K) \subseteq\left\{{\overline{U_{\alpha}}}^{c o c} \mid \alpha \in \Delta\left(y_{i}\right), i=1,2, \ldots, n\right\},
$$

hence $f^{-1}(K)$ is coc-weakly-compact relative to $X$.

Corollary 8. The coc-open coc-perfect function of a coc-weakly-compact space is cocweakly-compact.

Theorem 7. If $f: X \rightarrow Y$ is a coc-continuous surjective function and $X$ is coc- $R$ compact, then $Y$ is coc-R-compact. 
Proof. Let $\mathcal{U}=\left\{U_{\alpha} \mid \alpha \in \Delta\right\}$ be a coc-regular cover of $Y$. For each $\alpha \in \Delta$, there exists a coc-closed subset $F_{\alpha} \subseteq U_{\alpha}$ and $Y=\bigcup\left\{i n t_{c o c}\left(F_{\alpha}\right)\right\}$. Now $f^{-1}(\mathcal{U})=\left\{f^{-1}\left(U_{\alpha}\right) \mid \alpha \in \Delta\right\}$, $f^{-1}\left(F_{\alpha}\right) \subseteq f^{-1}\left(U_{\alpha}\right)$ and

$X=f^{-1}(Y)=f^{-1}\left(\bigcup\right.$ int $\left._{c o c}\left(F_{\alpha}\right)\right) \subseteq \bigcup f^{-1}\left(U_{\alpha}\right)=X$, clearly $f^{-1}(\mathcal{U})$ is coc-regular cover of $X$, so there exists a finite subcover such that

$$
X=\bigcup\left\{f^{-1}\left(U_{\alpha}\right)\left|\alpha \in \Delta_{0} \subseteq \Delta,\right| \Delta_{0} \mid<\omega_{0}\right\},
$$

hence the result.

Theorem 8. Let $f: X \rightarrow Y$ be a coc-almost coc-open and coc-perfect function. If $Y$ is coc-R-compact space, then $X$ is so.

Proof. Let $\mathcal{U}=\left\{U_{\alpha} \mid \alpha \in \Delta\right\}$ be a coc-regular cover of $X$, so for each $\alpha \in \Delta$, there exists a coc-closed subset $F_{\alpha} \subseteq U_{\alpha}$ and $X=\bigcup\left\{i n t_{c o c}\left(F_{\alpha}\right)\right\}$, but $f$ is coc-perfect function, so for each $y \in Y$, there is a finite set $\Delta_{0} \subseteq \Delta$ such that

$$
f^{-1}(y) \subseteq \bigcup_{\alpha \in \Delta_{0}} \operatorname{int}_{c o c}\left(F_{\alpha}\right) \subseteq \bigcup_{\alpha \in \Delta_{0}} F_{\alpha} \subseteq \bigcup_{\alpha \in \Delta_{0}} U_{\alpha} .
$$

Define

$$
\begin{aligned}
& K_{y}=Y-f\left(X-\bigcup\left\{i n t_{c o c}\left(F_{\alpha}\right) \mid \alpha \in \Delta_{0}\right\}\right), \\
& J_{y}=Y-f\left(X-{\overline{\bigcup\left\{i n t_{c o c}\left(F_{\alpha}\right) \mid \alpha \in \Delta_{0}\right\}}}^{c o c}\right) \text {, }
\end{aligned}
$$

and

$$
L_{y}=Y-f\left(X-\bigcup\left\{U_{\alpha} \mid \alpha \in \Delta_{0}\right\}\right) .
$$

Clearly $K_{y} \subseteq J_{y} \subseteq L_{y}$, also $K_{y}$ and $L_{y}$ are coc-open subsets of $Y$ and $J_{y}$ is coc-closed subset in $Y$, so for each $y \in Y$, there exists $\alpha \in \Delta_{0}$ such that $y \in K_{y}$, therefore $\left\{K_{y} \mid y \in Y\right\}$ is a coc-regular cover of $Y$, but $Y$ is coc-R-compact, so $Y=\bigcup\left\{L_{y_{i}} \mid i=1,2, \ldots, n\right\}$, moreover

$$
X=f^{-1}(Y)=f^{-1}\left(\left\{L_{y_{i}} \mid i=1,2, \ldots, n\right\}\right) \subseteq \bigcup\left\{U_{\alpha} \mid \alpha \in \Delta_{0}\right\},
$$

this complete the proof.

Corollary 9. Let $f: X \rightarrow Y$ be a coc-open coc-perfect coc-continuous, then $X$ is coc- $R$ compact space if and only if $Y$ is coc-R-compact space.

Corollary 10. If $X$ is coc-weakly-compact space and $Y$ is coc-compact space, then $X \times Y$ is coc-weakly-compact.

Proof. Notice that the projection $P_{X}: X \times Y \rightarrow X$ is coc-closed and the result comes from Theorem 6 .

Corollary 11. For a coc-almost-regular spaces $X$ and $Y$, if $X$ is coc-compact, then $X \times Y$ is coc-nearly (coc-weakly-compact, coc-R-compact).

Corollary 12. If $Y$ is coc-compact space, then $X \times Y$ is coc-almost-compact (coc-nearlycompact, coc-weakly-compact) if and only if $X$ is coc-R-compact space. 


\section{Acknowledgements}

The authors are grateful to the Middle East University, Amman, Jordan for the financial support granted to cover the publication fee of this research article.

\section{References}

[1] Z Altawalbeh. More on almost countably compact spaces. Italian Journal of Pure and Applied Mathematics, 43:177-184, 2020.

[2] Z Altawallbeh and A Al-Momany. Nearly countably compact spaces. International electronic journal of pure and applied Mathematics, 8(4):59-65, 2014.

[3] F Cammaroto. A note on weakly-compact spaces. Indian J. pure appl. Math, 16(12):1472-1477, 1985.

[4] F Cammaroto and G LO Faro. Weakly compact spaces. Riv. Mat. Univ. Parma, $7(4): 383-395,1981$.

[5] F Cammaroto and T Noiri. On r-compact spaces. MAT. BEC., 41:141-147, 1989.

[6] R Engelking. General Topology. Revised and completed edition. Heldermann Verlag, Berlin, 1989.

[7] Al Ghour, S, and S Samarah. Cocompact open sets and continuity. In Abstarct and Applied Analysis, P548612, 2012.

[8] C Kuratowski. Topologie I .4th edition, in French. Hanfner, New York, 1958.

[9] N Levine. Semi-open sets and semicontinuity in topological spaces. Amer. Math., 70:36-41, 1963.

[10] A Mashhour, M Abd El-Monsef, and S El Deeb. On precontinuous and weak precontinuous. Proc. Math. Phs. Soc. Egypt, 53:47-53, 1982.

[11] Al Ghour S and E Maghrabi. Co-compact separation axoims and slight co-continuity. Symmetry, 12, 2020.

[12] M Singal and S Arya. On almost-regular spaces. Glasnik Mat. Ser 3, 4:89-99, 1969. 